\title{
Fluorescence lifetime imaging microscopy of early $C$. elegans embryo development
}

Rupsa Datta ${ }^{1}$, Kelsey Tweed ${ }^{2}$ and Melissa Skala ${ }^{1}$

${ }^{1}$ Morgridge Institute for Research, Madison, Wisconsin, United States, ${ }^{2}$ University of WisconsinMadison, United States

During embryonic development, cells divide to form a functional body by establishing coordinated cell functions, fates, and behaviors. The energy demands vary at different stages of cell division and hence cellular metabolism can be highly dynamic during embryonic development both spatially and temporally [1]. Although evidence suggests a tight connection between rewiring of the cellular metabolic state and the progression of embryonic development, relatively little is known about dynamic transitions in metabolism during development. In this work we employ time-series fluorescence lifetime imaging microscopy (FLIM) of autofluorescent metabolic cofactor reduced nicotinamide adenine dinucleotide (phosphate), $\mathrm{NAD}(\mathrm{P}) \mathrm{H}$, to study the metabolic transitions in early Caenorhabditis elegans (C. elegans) embryogenesis. We chose $C$. elegans embryo as the model for this study due to its highly reproducible lineage and cellular morphology [2]. Moreover, C. elegans embryo undergoes asymmetric cell division that results in daughter cells with distinct cellular sizes, functions, and fates. Also, the anterior-posterior (AP) axis is established in the one-cell embryo (P0). The early embryo is only comprised of consecutive rounds of DNA synthesis ( $\mathrm{S}$ phase) and cell division during mitosis ( $\mathrm{M}$ phase), with no gap phase until the 28-cell stage [3]. Finally, genetic homogeneity can be achieved by self-fertilization of the hermaphrodites. NAD $(\mathrm{P}) \mathrm{H}$ is directly involved oxidation-reduction reactions in metabolic pathways and can be used to monitor the metabolism in a label-free manner. $\mathrm{NAD}(\mathrm{P}) \mathrm{H}$ in its free form has a relatively shorter fluorescence lifetime compared to when it is bound to proteins. Thus, FLIM of NAD(P)H provides insights into the metabolic state of cells [4]. We performed two-photon FLIM of live embryos undergoing divisions from the one-cell stage to four-cell stage. The lifetime data was acquired using time correlated single photon counting electronics and the fluorescence decay at each pixel was fit with a double exponential. NAD $(\mathrm{P}) \mathrm{H}$ lifetimes identified changes in metabolic states between embryonic cells with high temporal resolution. Specifically, NAD $(\mathrm{P}) \mathrm{H}$ lifetime significantly differed between $\mathrm{AB}$ and $\mathrm{P} 1$ cells which are blastomeres that result from the first asymmetric cell division in $C$. elegans embryo. We found that the $\mathrm{NAD}(\mathrm{P}) \mathrm{H}$ mean lifetime of $\mathrm{P} 1$ was significantly larger than $\mathrm{AB}$ indicating metabolic differences between the two blastomeres. Taken together this work demonstrates that two-photon FLIM of NAD $(\mathrm{P}) \mathrm{H}$ is a highly sensitive and non-invasive technique to study dynamic metabolic changes in early $C$. elegans embryo development.

\section{References}

1. H. Miyazawa and A. Aulehla, "Revisiting the role of metabolism during development," Development 145(19), (2018).

2. Z. Bao, Z. Zhao, T. J. Boyle, J. I. Murray, and R. H. Waterston, "Control of Cell Cycle Timing during C. elegans Embryogenesis," Dev Biol 318(1), 65-72 (2008).

3. M. L. Begasse and A. A. Hyman, "The First Cell Cycle of the Caenorhabditis elegans Embryo: Spatial and Temporal Control of an Asymmetric Cell Division," in Cell Cycle in Development, J. Z. Kubiak, ed., Results and Problems in Cell Differentiation (Springer Berlin Heidelberg, 2011), pp. 109-133.

4. R. Datta, T. M. Heaster, J. T. Sharick, A. A. Gillette, and M. C. Skala, "Fluorescence lifetime imaging microscopy: fundametals and advances in instrumentation, analysis, and applications," JBO 25(7), 071203 $(2020$ 